\title{
Use of Ebola Vaccine: Expansion of Recommendations of the Advisory Committee on Immunization Practices To Include Two Additional Populations — United States, 2021
}

\author{
Jason H. Malenfant, $\mathrm{MD}^{1,2}$; Allison Joyce, $\mathrm{MSc}^{2}$; Mary J. Choi, $\mathrm{MD}^{2}$; Caitlin M. Cossaboom, DVM, PhD²; Amy N. Whitesell, MPH²; \\ Brian H. Harcourt, $\mathrm{PhD}^{2}$; Robert L. Atmar, MD ${ }^{3}$; Julie M. Villanueva, $\mathrm{PhD}^{2}$; Beth P. Bell, MD ${ }^{4}$; Christine Hahn, MD ${ }^{5}$; Jamie Loehr, MD ${ }^{6}$; \\ Richard T. Davey, $\mathrm{MD}^{7}$; Armand Sprecher, $\mathrm{MD}^{8}$; Colleen S. Kraft, MD ; Trevor Shoemaker, $\mathrm{PhD}^{2}$; Joel M. Montgomery, PhD²; Rita Helfand, MD²; \\ Inger K. Damon, MD, $\mathrm{PhD}^{2}$; Sharon E. Frey, $\mathrm{MD}^{10}$; Wilbur H. Chen, $\mathrm{MD}^{11}$
}

\section{Introduction}

On December 19, 2019, the Food and Drug Administration (FDA) approved rVSV $\triangle \mathrm{G}-\mathrm{ZEBOV-GP}$ Ebola vaccine (ERVEBO, Merck) for the prevention of Ebola virus disease (EVD) caused by infection with Ebola virus, species Zaire ebolavirus, in adults aged $\geq 18$ years. In February 2020, the Advisory Committee on Immunization Practices (ACIP) recommended preexposure vaccination with ERVEBO for adults aged $\geq 18$ years in the United States who are at highest risk for potential occupational exposure to Ebola virus because they are responding to an outbreak of EVD, work as health care personnel at federally designated Ebola treatment centers in the United States, or work as laboratorians or other staff members at biosafety level 4 facilities in the United States (1).

This policy note reviews the expansion of these recommendations to include two additional populations: 1) health care personnel* involved in the care and transport of patients with suspected or confirmed EVD at special pathogens treatment centers (SPTCs) and 2) laboratorians and support staff members at Laboratory Response Network (LRN) facilities that handle specimens that might contain replication-competent Ebola virus (species Zaire ebolavirus) in the United States.

\section{Background}

Ebola virus, species Zaire ebolavirus, is the most lethal of the four viruses that cause EVD in humans, with case fatality rates of $70 \%-90 \%$ when untreated (2). The virus is highly transmissible and can be found in all body fluids of an infected

\footnotetext{
* Health care personnel refers to all paid and unpaid persons serving in health care settings who have the potential for direct or indirect exposure to patients or infectious materials, including body substances (e.g., blood, tissue, and specific body fluids); contaminated medical supplies, devices, and equipment; contaminated environmental surfaces; or contaminated air. These health care personnel include, but are not limited to, emergency medical service personnel, nurses, nursing assistants, physicians, technicians, clinical laboratory personnel, autopsy personnel, therapists, phlebotomists, pharmacists, students and trainees, contractual staff members not employed by the health care facility, and persons not directly involved in patient care, but who could be exposed to infectious agents that can be transmitted in the health care setting (e.g., clerical, dietary, environmental services, laundry, security, engineering and facilities management, administrative, billing, and volunteer personnel).
}

person (3-5). If untreated, death from EVD can be rapid, usually occurring 7-10 days after the onset of symptoms (6-9).

\section{Methods}

During March 2020-November 2021, the Ebola Vaccine Work Group met at least monthly via conference call to review and discuss relevant evidence regarding expansion of recommendations to the two populations of interest using the Evidence to Recommendations framework. ${ }^{\dagger}$

SPTCs. SPTCs (formerly known as state-designated Ebola treatment centers) are health care facilities, designated by states, that intend to receive and can provide care for a patient with suspected or confirmed EVD for the duration of their illness (10). Currently, there are approximately 55 U.S. SPTCs, with 100-150 health care personnel at each facility. Upon the recommendation of the Council for State and Territorial Epidemiologists, the name "special pathogens treatment centers" replaced "state-designated Ebola treatment centers" because many of these centers have the capability to treat patients with other diseases in addition to EVD.

LRN facilities. LRN is a large network of laboratories throughout the United States; these facilities aim to rapidly respond to biologic and chemical threats and other public health emergencies. Within the LRN, there are currently 58 laboratories that have the capacity to test for Ebola virus, with up to 15 persons at each facility trained to perform the testing (11).

Knowledge, Attitude and Practices survey. A Knowledge, Attitude and Practices survey was distributed to personnel at both SPTCs and LRN facilities. The purpose of the survey was to measure EVD vaccine acceptability and sentiments in these populations. Survey questions assessed perceived severity of EVD and risk for infection, interest in receiving the vaccine, and concerns about the vaccine. SPTCs and LRN facilities were provided anonymous survey website links to a point of contact at each site. The survey was distributed to the SPTCs on October 14, 2020 and to LRN facilities on

\footnotetext{
$\dagger^{\dagger}$ https://www.cdc.gov/vaccines/acip/recs/grade/ebola-vaccine-etr.html
} 
December 29, 2020 and remained open for both populations until January 22, 2021. $\$$

Vaccine efficacy and safety. The Ebola Vaccine Work Group relied upon Grading of Recommendations, Assessment, Development and Evaluation (GRADE) evidence profiles, which provide detailed methods and results used to assess Ebola vaccine efficacy and safety. These profiles were presented at the February 26, 2020, ACIP meeting.

\section{Summary of Major Findings}

Knowledge, Attitude and Practices survey to SPTCs and LRN facilities. Fifty-one SPTCs in 24 states were identified at the time of the survey. Among those, the survey was distributed to 49 centers; contact information was missing for two of the centers. In total, 364 survey responses were received from the SPTC population; 66 were excluded because of incompleteness, leaving 298 responses for analyses. Among SPTC respondents, $69 \%$ were women and $52 \%$ were aged $\geq 40$ years. Thirty-nine percent of SPTC respondents self-identified as nurses and $22 \%$ as physicians. Additional health care professional groups included respiratory therapists, emergency medical technicians, advanced practice providers, laboratory technicians, and others. Sixty-two LRN facilities (the number of facilities capable of testing for Ebola virus at the time of the survey) were identified. Ninety-six survey responses were received from this population; 26 were excluded because of incompleteness, leaving 70 responses included for analyses. Among LRN respondents, $64 \%$ were women and $76 \%$ were aged $\geq 40$ years. Most LRN respondents (64\%) self-identified as "laboratory scientist."

Fifty-four percent of SPTC survey respondents reported willingness to be vaccinated if they were eligible and offered the vaccine at the time of survey administration. ${ }^{* *}$ When given the choice to get vaccinated at different time points (i.e., when an EVD case is imported to the United States or when an EVD case is imported to their state), willingness to receive vaccine increased to $81 \%$. When asked whether they believed that ACIP should recommend the vaccine for their population, $53 \%$ responded yes, $9 \%$ responded no, and $38 \%$ were unsure.

Fifty-nine percent of LRN survey respondents reported willingness to be vaccinated if they were eligible and offered the vaccine at the time of survey administration. ${ }^{\dagger \dagger}$ When given the

\footnotetext{
$\S$ During this time, there was an Ebola outbreak in Equateur Province in the Democratic Republic of the Congo; the end of the outbreak was declared on November 18, 2020.

https://www.cdc.gov/vaccines/acip/recs/grade/ebola-vaccine.html

** These respondents took the survey during October 14, 2020-January 22, 2021. During this time, there was an Ebola outbreak in Equateur Province in the Democratic Republic of the Congo; the end of the outbreak was declared on November 18, 2020.

†† These respondents took the survey during December 29, 2020-January 21, 2021. During this time, there were no active Ebola virus outbreaks in the world.
}

\begin{abstract}
Summary
What is already known about this topic?

Preexposure vaccination against Ebola virus disease is currently recommended for adults aged $\geq 18$ years in the United States who are at highest risk for occupational exposure to Ebola virus.

What is added by this report?

The Advisory Committee on Immunization Practices has expanded recommendations to include two additional populations at high risk for potential occupational exposure to Ebola virus: health care personnel at special pathogens treatment centers and laboratorians and support staff members at Laboratory Response Network facilities.

What are the implications for public health practice?

Ebola virus remains an international and domestic public health threat. Preexposure vaccination can protect those at occupational risk for exposure to Ebola virus.
\end{abstract}

choice to get vaccinated at different time points (i.e., when an EVD case is imported to the United States, or when an EVD case is imported to their state), willingness to receive vaccine increased to $86 \%$. When asked whether they believed that ACIP should recommend the vaccine for their population, $59 \%$ responded yes, $9 \%$ responded no, and $33 \%$ were unsure.

\section{Rationale}

Similar to the initial groups for whom the vaccine was recommended in February 2020, the decision to recommend preexposure vaccination in these groups was based on the following conditions: 1) documented protective efficacy of the vaccine against the development of symptomatic EVD, 2) high mortality and severity of illness in persons infected with Ebola virus, 3) high transmissibility of Ebola virus, 4) EVD-related sequelae in survivors, 5) the potential for continued disease transmission and disease recrudescence, and 6) an acceptable safety profile relative to the severity of Ebola virus infection.

\section{Recommendations}

Preexposure vaccination with ERVEBO is now also recommended for adults aged $\geq 18$ years in the U.S. population who are at high risk for potential occupational exposure to Ebola virus:

- Health care personnel involved in the care and transport of patients with suspected or confirmed EVD at SPTCs, or

- Laboratorians and support staff members at LRN facilities that handle specimens that might contain replicationcompetent Ebola virus (species Zaire ebolavirus) in the United States. 


\section{Future Research and Monitoring Priorities}

Research regarding ERVEBO is ongoing, including safety of ERVEBO in immunocompromised persons, pregnant women, and children. The same considerations and recommendations for these special populations apply as in the initial recommendations (1). In addition, long-term studies are continuing to assess immunogenicity and duration of protection. ACIP will consider these data as they become available and revise recommendations accordingly.

\section{Acknowledgments}

Members of the Advisory Committee on Immunization Practices; member roster as of November 2021 is available (https://www.cdc. gov/vaccines/acip/members/index.html).

Corresponding author: Jason H. Malenfant, rix3@cdc.gov.

\footnotetext{
${ }^{1}$ Epidemic Intelligence Service, CDC; ${ }^{2}$ Division of High Consequence Pathogens and Pathology, National Center for Emerging and Zoonotic Infectious Diseases, CDC; ${ }^{3}$ Baylor College of Medicine, Houston, Texas; ${ }^{4}$ University of Washington, Seattle, Washington; ${ }^{5}$ Idaho Department of Health and Welfare; ${ }^{6}$ Cayuga Family Medicine, Ithaca, New York; ${ }^{7}$ National Institute of Allergy and Infectious Diseases, Bethesda, Maryland; ${ }^{8}$ Médicins Sans Frontières, Brussels, Belgium; ${ }^{9}$ Emory University, Atlanta, Georgia; ${ }^{10}$ Saint Louis University School of Medicine, St. Louis, Missouri; ${ }^{11}$ University of Maryland School of Medicine, Baltimore, Maryland.
}

All authors have completed and submitted the International Committee of Medical Journal Editors form for disclosure of potential conflicts of interest. No potential conflicts of interest were disclosed.

\section{References}

1. Choi MJ, Cossaboom CM, Whitesell AN, et al. Use of Ebola vaccine: recommendations of the Advisory Committee on Immunization Practices, United States, 2020. MMWR Recomm Rep 2021;70:1-12. PMID:33417593 https://doi.org/10.15585/mmwr.rr7001a1

2. Feldmann H, Geisbert TW. Ebola haemorrhagic fever. Lancet 2011;377:849-62. PMID:21084112 https://doi.org/10.1016/ S0140-6736(10)60667-8

3. Bausch DG, Towner JS, Dowell SF, et al. Assessment of the risk of Ebola virus transmission from bodily fluids and fomites. J Infect Dis 2007;196(Suppl 2):S142-7. PMID:17940942 https://doi. org/10.1086/520545

4. Dowell SF, Mukunu R, Ksiazek TG, Khan AS, Rollin PE, Peters CJ. Transmission of Ebola hemorrhagic fever: a study of risk factors in family members, Kikwit, Democratic Republic of the Congo, 1995. Commission deLuttecontreles Epidémiesà Kikwit.J Infect Dis 1999;179(Suppl 1):S87-91. PMID:9988169 https://doi.org/10.1086/514284

5. Sissoko D, Keïta M, Diallo B, et al. Ebola virus persistence in breast milk after no reported illness: a likely source of virus transmission from mother to child. Clin Infect Dis 2017;64:513-6. PMID:27940938

6. Malvy D, McElroy AK, de Clerck H, Günther S, van Griensven J. Ebola virus disease. Lancet 2019;393:936-48. PMID:30777297 https://doi. org/10.1016/S0140-6736(18)33132-5

7. Kortepeter MG, Bausch DG, Bray M. Basic clinical and laboratory features of filoviral hemorrhagic fever. J Infect Dis 2011;204(Suppl 3):S810-6. PMID:21987756 https://doi.org/10.1093/ infdis/jir299

8. Liddell AM, Davey RT Jr, Mehta AK, et al. Characteristics and clinical management of a cluster of 3 patients with Ebola virus disease, including the first domestically acquired cases in the United States. Ann Intern Med 2015;163:81-90. PMID:25961438 https://doi.org/10.7326/ M15-0530

9. Bah EI, Lamah MC, Fletcher T, et al. Clinical presentation of patients with Ebola virus disease in Conakry, Guinea. N Engl J Med 2015;372:40-7. PMID:25372658 https://doi.org/10.1056/ NEJMoa1411249

10. CDC. Interim guidance for preparing Ebola treatment centers. Atlanta, GA: US Department of Health and Human Services, CDC; 2015. https://www.cdc.gov/vhf/ebola/healthcare-us/preparing/treatmentcenters.html

11. CDC. The laboratory response network partners in preparedness. Atlanta, GA: US Department of Health and Human Services, CDC; 2019. https://emergency.cdc.gov/lrn/ 